\title{
FLORID CEMENTO-OSSEOUS DYSPLASIA AS A POSSIBLE AGGRAVATING FACTOR IN A CASE OF TRIGEMINAL NEURALGIA
}

C.H. Siar, N. Ibrahim, A.N. Omar, Z.A. Abdul Rahman. Florid cemento-osseous dysplasia as a possible aggravating factor in a case of trigeminal neuralgia. Annal Dent Univ Malaya 2010; 17: 21-24.

\begin{abstract}
Differential diagnosis of orofacial pain is crucial, as the course of each process and its clinical management varies markedly. A case is illustrated here of trigeminal neuralgia in a 49-year-old Indian female whose complaint was initially diagnosed as dental pain leading to sequential extractions of her right mandibular and maxillary molars but with no pain abatement. Subsequent neurological assessment diagnosed her complaint as trigeminal neuralgia but pain remained poorly controlled even with high doses of carbamazepine and gabapentin. A dental referral and orthopantomographic examination revealed multifocal sclerotic masses in her jaws, suggestive of florid cemento-osseous dysplasia (FCOD). Right mandibular incisional biopsy confirmed the diagnosis. A decision was made to curette the right mandibular masses and lateralised the right inferior dental nerve. Follow-up disclosed considerable pain reduction. This case raises the issue as to whether the sclerotic bone masses in FCOD may have caused nerve compression which aggravated her neuralgic pain.
\end{abstract}

Key words: trigeminal neuralgia; florid cementoosseous dysplasia; pain

\section{INTRODUCTION}

A wide and diverse range of painful maladies occur in the orofacial region (1). Trigeminal neuralgia is a neuropathic condition characterized by unilateral short-lasting, lancinating, electric-shock-type pain that arises from one or more branches of the trigeminal nerve $(2,3)$. The precise mechanism underlying this pain disorder remains unclear (4). Among the numerous theories put forth (1-4), the microvascular compression aetiological concept is the most widely accepted. Accordingly, vascular compression of nerve roots by tortuous vessels causes segmental demyelination of the affected nerve which in turn leads to nonsynaptic or ephaptic transmission of nerve impulses (2-5). Clinically, trigger points and innocuous triggering stimuli such as touch, chewing, talking may be identified. Current diagnostic criteria recognized two distinct subsets of trigeminal neuralgia: classical/ idiopathic/primary form which accounts for vast majority of trigeminal neuralgia and symptomatic/
Original Article

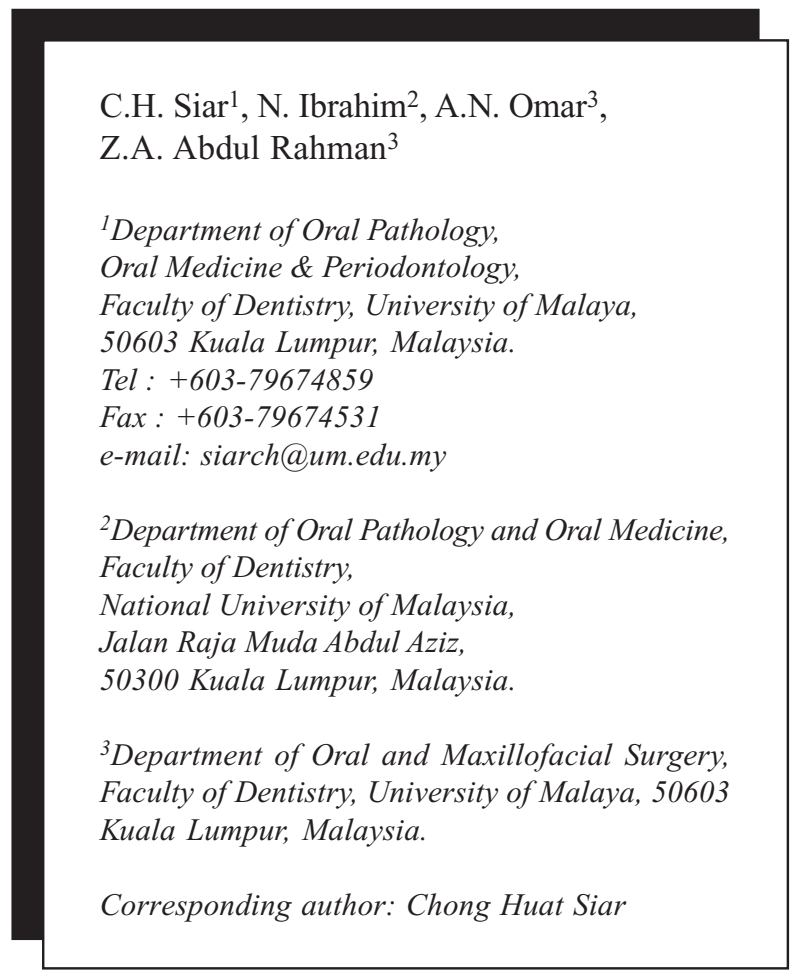

secondary variant which occurs secondary to microvascular compression, intracranial tumours or multiple sclerosis (2-4). A third form unrecognized by current classification is atypical trigeminal neuralgia which accounts for up to a third of these cases.

Cemento-osseous dysplasia is the most common type of fibro-osseous lesion of the maxillofacial region (6). It represents a reactive process in which normal bone is replaced by a poorly cellularized connective tissue with cemento-osseous deposits. Florid cementoosseous dysplasia, a distinct subset of cementoosseous dysplasia, is characterized by multifocal lesions in the tooth bearing or edentulous areas of the maxilla and mandible, often distributed in a bilateral symmetric manner (6-9). This disorder shows a $90 \%$ female predominance, a strong predilection for black patients, and a mean onset age of 42 years. As with the other subtypes, the lesions demonstrate three stages of maturation, and most patients are asymptomatic, though some may complain of dull pain, sinus tracts, or exposure of avascular bone into the oral cavity due to secondary infection (9).

\section{CASE REPORT}

History of present complaint: A 49-year-old Indian female patient, a known case of trigeminal neuralgia, was referred to the Oral Medicine Clinic for management of severe pain affecting her right upper 
and lower jaws. The pain was episodic and of a sharp shooting nature. It started along the right side of the mandible and extended to the right face and right ear. Aggravating factors included talking, chewing and washing of her face. Pain also disturbed her sleep.

Past dental history: According to the patient, her pain started more than 22 years ago in the right posterior mandibular region. This was diagnosed as pain of odontogenic origin which led to sequential extraction of her right mandibular premolars and molars followed by her right maxillary molars. However there was no improvement in her pain complaint.

Past medical history: Subsequent neurological assessment diagnosed her pain complaint as trigeminal neuralgia. She was placed on a combination of gabapentin at a starting dose of $100 \mathrm{mg} x$ od titrated to $900 \mathrm{mg} \mathrm{x} \mathrm{od,} \mathrm{and} \mathrm{carbamazepine} \mathrm{(200} \mathrm{mg} \mathrm{x} \mathrm{bd).}$ Recently, this regimen did not control her pain.

Oral Surgery referral: She was then referred to the Oral Surgery Department here for further management. At presentation, there was a slight swelling of her right mandible which was painful on touching. There was no regional lymphadenopathy. Orthopantomogram revealed presence of patchy areas of mixed radiolucencies and radiopacities affecting all four quadrants of her jaws (Fig. 1). Differential radiologic diagnoses given included focal sclerosing osteomyelitis, cementoblastoma and hypercementosis.

Biopsy: Under local anaesthesia, an incisional biopsy was performed in the right mandibular lesions for histopathological examination. Microscopic evaluation revealed fibrocellular tissues with scattered rounded sclerotic cemento-osseous masses of varying sizes (Fig. 2). The fibrocellular component was cytologically bland-looking.

Treatment and follow-up: A decision was made to curette the right mandibular sclerotic masses and lateralized the right inferior dental nerve. Follow-up revealed that her pain severity has reduced considerably. She was able to achieve good pain control $\mathrm{t}$ with gabapentin $600 \mathrm{mg} \mathrm{x}$ od and tegretol $200 \mathrm{mg} x$ od. However no long-term follow-up information was available as to the outcome of this procedure as the patient failed her subsequent review appointments.

\section{DISCUSSION}

Diagnosis and treatment of trigeminal neuralgia remains challenging. Lack of objective clinical tests, and overlapping signs and symptoms with other orofacial pain disorders are the two key reasons for the frequent misdiagnosis of trigeminal neuralgia (10-14). In most instances including the current case, trigeminal neuralgia was misdiagnosed as dental pain leading to sequential loss of the teeth in the complaint site (10, 13, 14).

Florid cemento-osseous dysplasia is mostly an asymptomatic process with dull pain in those cases presenting with exposure of the sclerotic masses into the oral cavity either due to progressive alveolar bone atrophy under a denture or following tooth extractions in the affected area (6-8). Neurovascular compression is a known cause of trigeminal neuralgia (2-4). The most significant finding of this case was pain reduction postoperatively following surgical curettage of the cemento-osseous lesions and lateralization of the inferior dental nerve in the right mandible. We speculated that the sclerotic masses that occurred in florid cemento-osseous dysplasia may have caused chronic compression of the inferior dental nerve leading to either an exuberant neural response in the form of a traumatic neuroma formation or degenerative changes and demyelination, both of which may manifest as severe neuralgic pain. Neural compression by vascular structures is the most widely accepted

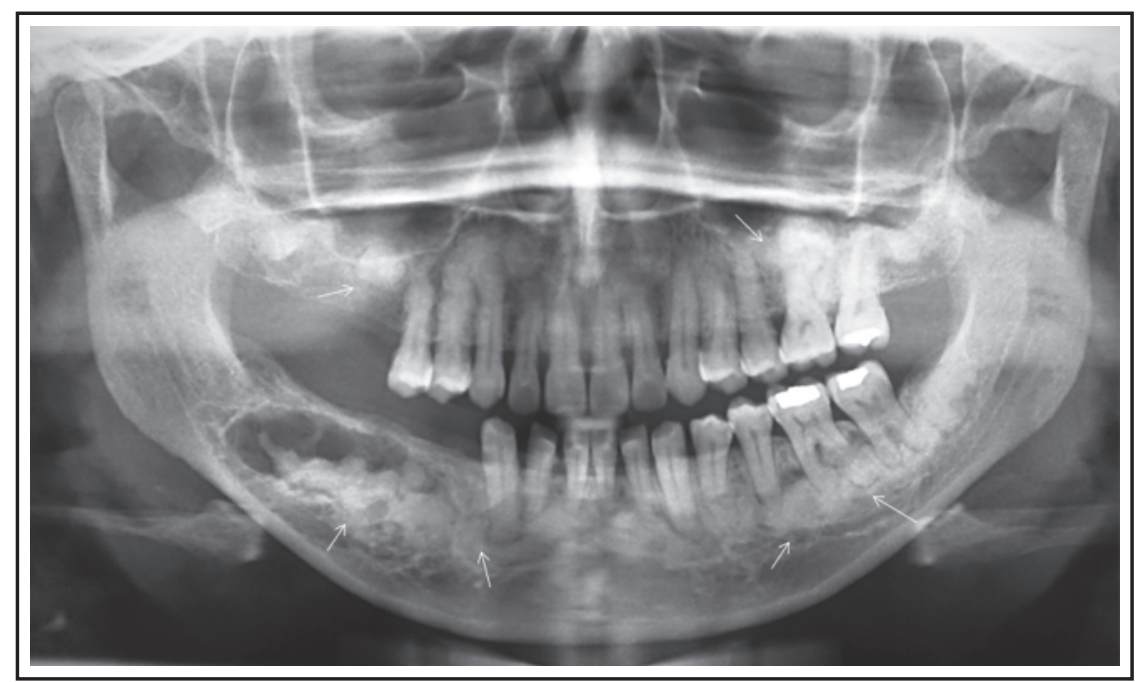

Figure 1. Orthopantomogram showing multifocal sclerotic masses (arrows) in all four quadrants of the jaws. 


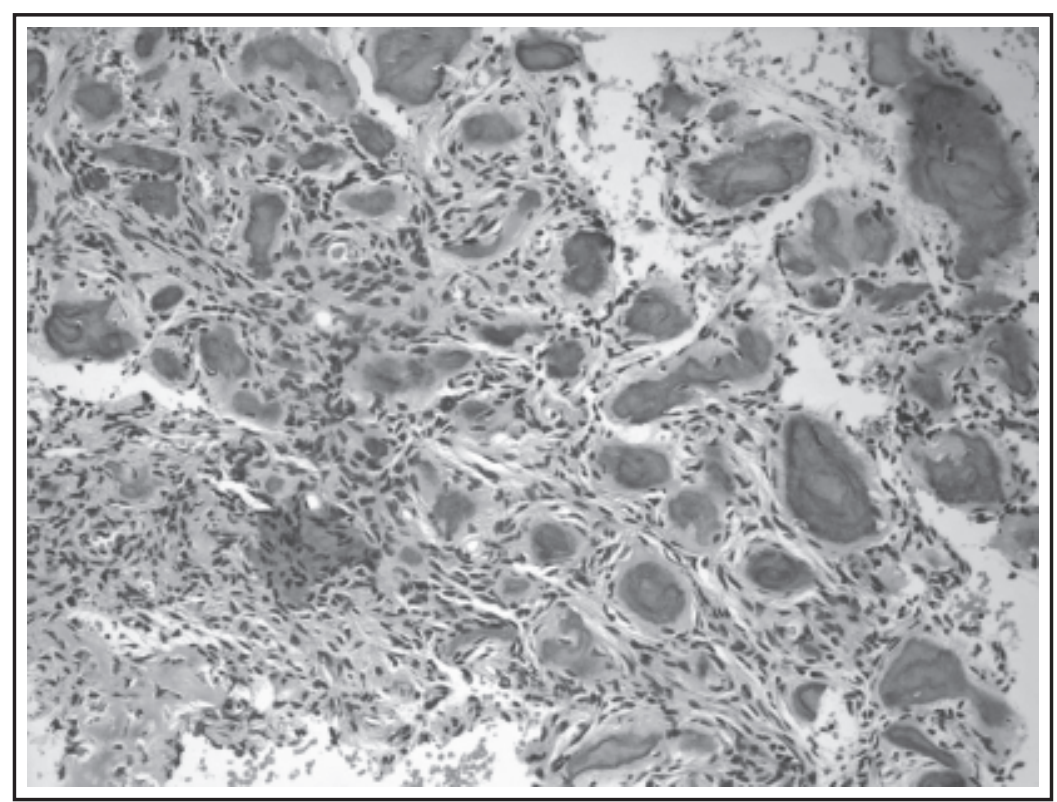

Figure 2. Photomicrograph shows fibrocellular tissue with scattered spheroidal cemento-osseous deposits. (HE, Original magnification, x 100).

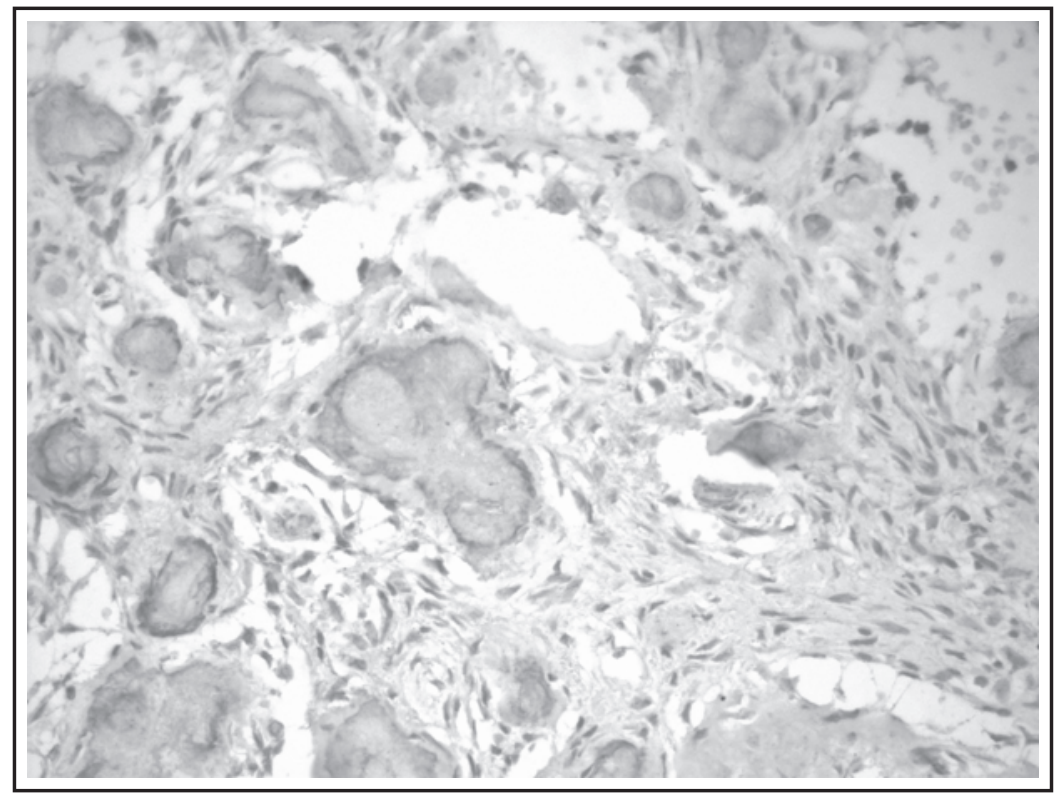

Figure 3. Immunohistochemical staining did not show any neural proliferation within the lesional area (anti-S-100 protein, Original magnification x100).

theory underlying the pain mechanism in trigeminal neuralgia. In this case, the assumed cause of the nerve compression was the sclerotic cemento-osseous masses. However this speculation was unsubstantiated. Serial sectioning studies aided by limited immunohistochemical staining for nerve markers (neuron specific enolase and S-100 protein) of the bone currettings did not show any evidence of neural proliferation within the lesional tissues of florid cemento-osseous dysplasia (Fig. 3). Furthermore, when we reviewed our archives for cases of trigeminal neuralgia treated at the Oral Medicine Clinic ( $\mathrm{SCH}$ cases), only one other patient presenting with simultaneous occurrence of trigeminal neuralgia and florid cemento-osseous dysplasia was encountered. However because of her good pain control with carbamazepine (200mg three times daily) alone, no surgical intervention to remove the cemento-osseous lesions was attempted.

Repositioning of the inferior dental nerve is not an uncommon surgical procedure (15-17). There are two basic approaches: fenestration and lateralization. Fenestration involves creating a cortical bone window posterior to the mental foramen along the length of 
the canal to free the inferior dental nerve and repositioning it after placement of, for example, endosseous implants (15). For lateralization, an osteotomy is performed around the mental nerve foramen extending posteriorly with a window until the nerve is fully freed from the intrabony trajectory (15). This later technique was employed to reposition the right inferior dental nerve in the present case. No bone grafting and avoidance of donor site morbidity are amongst the advantages with this procedure.

In summary, the case presented here serves to illustrate that it is important for clinicians to consider trigeminal neuralgia as a differential diagnosis in patients presenting with paroxysms of pain. This is so as to obviate from unwarranted treatments such as extractions, and that the patients can be referred early on for definitive therapy.

\section{REFERENCES}

1. Koopman JS, Dieleman JP, Huygen FJ, de Mos M, Martin CG, Sturkenboom MC. Incidence of facial pain in the general population. Pain 2009; 147:122-7.

2. Zakrzewska JM. Assessment and treatment of trigeminal neuralgia. Br J Hosp Med 2010;71: 490-4

3. McLeod NMH, Tekeli KM, Cheriyan J. Trigeminal neuralgia: assessment and management by oral and maxillofacial surgeons in the United Kingdom. $\mathrm{Br}$ J Oral Maxillofac Surg 2009;47:42-5.

4. Jia D, Gang L. Bioresonance hypothesis: A new mechanism on the pathogenesis of trigeminal neuralgia. Med Hypothesis 2010;74:505-7.

5. Toda K. Operative treatment of trigeminal neuralgia: review of current techniques. Oral Surg Oral Med Oral Pathol Oral Radiol Endod 2008; 106:788-805.

6. Barnes L, Eveson JW, Reichart P, Sidransky D ed. World Health Organization Classification of Tumours. 2005, IARC Press, Lyon, France.

7. Waldron CA, Giansanti JS, Browand BC. Sclerotic cemental masses of the jaws (so-called chronic sclerosing osteomyelitis, sclerosing osteitis, multiple enostosis, and gigantiform cementoma. Oral Surg Oral Med Oral Pathol 1975;39:590604.
8. Schneider LC, Mesa ML. Differences between florid osseous dysplasia and chronic diffuse sclerosing osteomyelitis. Oral Surg Oral Med Oral Pathol 1990;70:308-12.

9. Groot RH, van Merkesteyn JP, Bras J. Diffuse sclerosing osteomyelitis and florid osseous dysplasia. Oral Surg Oral Med Oral Pathol Oral Radiol Endod 1996;81:333-42.

10. Spencer CJ, Neubert JK, Gremillion H, Zakrzewska JM. Toothache or trigeminal neuralgia: Treatment dilemmas. J Pain 2008;9:767-70.

11. Sato J, Saitoh T, Notani K, Fukuda H, Kaneyama K, Segami N. Diagnostic significance of carbamazepine and trigger zones in trigeminal neuralgia. Oral Surg Oral Med Oral Pathol Oral Radiol Endod 2004;97:18-22.

12. Lewis MAO, Sankar V, De Laat A, Benliel R. Management of neuropathic orofacial pain. Oral Surg Oral Med Oral Pathol Oral Radiol Endod 2007;103:S32:e1 - e24.

13. de Siqueira SR, Nobrega JC, Valle LB, Teixeira MJ, de Siqueira JT. Idiopathic trigeminal neuralgia: clinical aspects and dental procedures. Oral Surg Oral Med Oral Pathol Oral Radiol Endod 2004; 98:311-5

14. Law AS, Lilly JP. Trigeminal neuralgia mimicking odontogenic pain. A report of two cases. Oral Surg Oral Med Oral Pathol Oral Radiol Endod 1995; 80:96-100.

15. Del-Castillo-Pardo-de-Vera JL, Chamorro M, Cebrián-Carretero JL. Repositioning of the inferior dental nerve in cases of severe mandibular atrophy. A clinical case. Med Oral Patol Oral Cir Bucal 2008;13:E778-82.

16. Alling CC. Lateral repositioning of inferior alveolar neurovascular bundle. J Oral Surg. 1977;35:419.

17. Jensen O, Nock D. Inferior alveolar nerve repositioning in conjunction with placement of osseointegrated implants: a case report. Oral Surg Oral Med Oral Pathol. 1987;63:263-8. 